\title{
Cardiac arrhythmias in low- and middle-income countries
}

\author{
Philasande Mkoko', Ehete Bahiru², Olujimi A. Ajijola ${ }^{2}$, Aime Bonny ${ }^{3,4}$, Ashley Chin ${ }^{1}$ \\ ${ }^{1}$ The Cardiac Clinic, Department of Medicine, Groote Schuur Hospital and University of Cape Town, Cape Town, South Africa; ${ }^{2}$ UCLA Cardiac \\ Arrhythmia Center, David Geffen School of Medicine at UCLA, Los Angeles, CA, USA; ${ }^{3}$ Department of internal medicine, District hospital \\ Bonassama, University of Douala, Douala, Cameroon; ${ }^{4}$ Service de cardiologie, Hôpital Forcilles, Ferolles-Attilly, France, Unité de rythmologie, \\ Centre hospitalier Le Raincy-Montfermeil, Montfermeil, France \\ Contributions: (I) Conception and design: P Mkoko, A Chin; (II) Administrative support: All authors; (III) Provision of study materials or patients: \\ All authors; (IV) Collection and assembly of data: All authors; (V) Data analysis and interpretation: All authors; (VI) Manuscript writing: All \\ authors; (VII) Final approval of manuscript: All authors. \\ Correspondence to: Ashley Chin. The Cardiac Clinic, Department of Medicine, Groote Schuur Hospital and University of Cape Town, Cape Town, \\ South Africa. Email: Ashley.chin@uct.ac.za.
}

\begin{abstract}
Many low- and middle-income countries (LMICs) are undergoing an epidemiological transition. With an improvement in socioeconomic conditions and an aging population, cardiovascular diseases (CVDs), like cardiac arrhythmias, are expected to increase in these countries. However, there are limited studies on the epidemiology and management of cardiac arrhythmias in LMICs. This review will highlight the unique challenges and opportunities that these countries face when managing cardiac arrhythmias.
\end{abstract}

Keywords: Arrhythmias; developing world; low- and middle-income countries (LMICs); pacing; atrial fibrillation (AF); tachycardias; bradycardias

Submitted Aug 13, 2019. Accepted for publication Sep 26, 2019.

doi: $10.21037 / \mathrm{cdt} .2019 .09 .21$

View this article at: http://dx.doi.org/10.21037/cdt.2019.09.21

\section{Introduction}

The majority of the world's low- and middle-income countries (LMICs) are undergoing an epidemiological transition. With an improvement in socioeconomic conditions, the epidemiological transition has led to the emergence of non-communicable diseases especially cardiovascular diseases (CVDs) (1). CVDs are responsible for the leading causes of death globally. In 2016, more than $75 \%$ of the 17.9 million deaths from CVDs occurred in LMICs (2). Ischaemic heart disease and cerebrovascular disease are responsible of the vast majority of cardiovascular deaths in LMICs (2).

While intensive global efforts to prevent and treat cardiovascular deaths in high income countries (HICs) and LMICs are underway, cardiac arrhythmias remain a neglected group of CVDs, especially in LMICs. With an increasing life expectancy seen in most LMICs, arrhythmias like atrial fibrillation (AF), ventricular arrhythmias due to ischaemic heart disease, sinus node dysfunction (SND) and heart block are expected to increase in these countries. As the treatment of cardiac arrhythmias often requires specialized skills, facilities, expensive equipment and devices to treat them effectively, it is not surprising that a wide disparity exists in the standard of arrhythmia care between HICs and LMICs.

This review article will focus on the current epidemiology and discuss the challenges to the management of cardiac arrhythmias and opportunities to improve arrhythmia care in LMICs.
AF
Epidemiology
$\mathrm{AF}$ is the most common arrhythmia globally. The 2010 Global Burden of Disease (GBD) data estimated the global burden of AF at 33.5 million (3). In HICs, such as the United States, AF affects 2-6 million people currently and it is expected to double by $2060(4,5)$. The prevalence 
and incidence of AF in HICs remains significantly higher compared to LMICs; however, this difference needs to factor in the substantial lack of data from LMICs that likely significantly underestimates the true disease burden $(3,4)$. In the $2010 \mathrm{GBD}$ study, the estimated AF prevalence per 100,000 population in LMICs was 565.7 (95\% CI, 522.9617.6) among men and 366.1 (337.4-400.8) among women and the estimated incidence rates per 100,000 person-years were 53.0 (33.8-76.8) among men and 40.0 (27.2-62.6) among women (3).

A systematic review of community-based AF epidemiology studies in LMICs showed a prevalence of $0.03-1.25 \%$ in the general population and $0.67-5.6 \%$ in individuals older than 70 years of age (6). A systematic review assessing the epidemiology of AF in Asia based on 58 studies from 8 countries showed an annual incidence of 5.38 per 1,000 person-years with the prevalence ranging from $0.37-15.8 \%$ across community and hospital-based studies (7). The study also found the use of warfarin to be less than $50 \%$ in hospital-based studies (7). A primary carebased retrospective observational study including 262,685 primary care patients in Brazil found the overall prevalence of $\mathrm{AF}$ to be $1.8 \%$, which is equivalent to other HICs, with a very low use of anticoagulation at $1.5 \%$ (8). Another study using data from seven Latin American countries, both in the inpatient and outpatient settings, found a $1.6 \%$ total prevalence of $\mathrm{AF}$ with $85.9 \%$ of cases being non-valvular $\mathrm{AF}$ and $18-25 \%$ of the those diagnosed with $\mathrm{AF}$ were not on any anticoagulant therapy (9). The epidemiology of AF in SSA is also not very well defined due to lack of largescale population wide studies from the region. However, smaller scale hospital or community-based studies have shown a prevalence ranging from $0.7-5.5 \%$ (10).

$\mathrm{AF}$ patients in LMICs tend to present younger and in heart failure compared to HICs and tend to have high prevalence of stroke at $10-27 \%$ with severe and debilitating symptoms and are less likely to receive guideline directed management $(6,11)$. A sub-analysis from the Randomized Evaluation of Long-Term Anticoagulation Therapy (RE-LY) registry showed large regional variabilities in the prevalence of $\mathrm{AF}$, risk factors, and anticoagulation management from data representing 46 countries and all income categories. The study found the prevalence of $\mathrm{AF}$ to be lower in LMICs compared to HICs, and patients with non-valvular AF on average were 7 years younger in comparison (12). One-year outcomes from the RELY registry showed mortality rates ranging from $17-20 \%$ in South America and Africa compared to the 10\% mortality rates in North America, western Europe, and Australia (13). There is also a marked gender disparity in mortality associated with AF in LMICs with women being disproportionately affected compared to men (3). Two-year clinical outcomes of AF based on the Global Anticoagulant Registry in the FIELD-Atrial Fibrillation (GARFIELD-AF) observational study between 2010-2016 including 17,162 patients from 30 countries found that mortality was the most frequent adverse event with rates of all-cause mortality at 3.83 (95\% CI, 3.62-4.05) per 100 person-years and cardiovascular death was $1.55(1.42-1.70)$ per 100 personyears (14).

\section{Risk factors}

Traditional CVD risk factors including aging, diabetes, obesity and particularly hypertension have been identified as significant drivers of the overall growing AF burden globally $(3,4,6)$. Improvements in life expectancies and urbanization are accelerating the growing burden of traditional CVD risk factors in LMICs and will also contribute to the growing burden of AF. Valvular heart diseases including rheumatic heart disease (RHD), which has a high prevalence in LMICs, are also risk factors for AF (6). Based on the RE-LY registry, hypertension was found to be the most common risk factor globally (12). However, RHD, which had a prevalence of $21.5 \%$ in Africa and $31.5 \%$ in India among the included patients, was a significant risk factor in these regions (12).

\section{Challenges to management of AF in LMICs}

There are several challenges to the optimal management of AF in LMICs. Optimal management of AF is based on international guidelines including the 2014 American Heart Association/American College of Cardiology/ Heart Rhythm Society (AHA/ACC/HRS) $(15,16)$ and the 2016 European Society of Cardiology (ESC) practice guidelines (17). The true burden of the disease and current management trends are not well understood in most LMICs (3-6). LMICs often lack strong health care systems designed to adequately respond to the rising burden of CVDs including AF. There are significant limitations to the proper implementation of current international AF management guidelines. First, most international guidelines are based on evidence generated in high income settings and tailored to healthcare systems with adequate resources and capability to implement time-sensitive complex interventions (18). The 
applicability of these widely accepted guidelines to resource limited settings are not well understood. The World Heart Federation (WHF) identifies three main areas of care gaps in the management of $\mathrm{AF}$ in LMICs including inadequate screening for $\mathrm{AF}$ among high risk individuals and those with non-valvular AF that may not have significant symptoms, low rates of initiation on anticoagulation, and the lack of country specific AF data to tailor interventions particularly in LMIC where the extent of care gap is highly variable (19). The roadblocks highlighted by the WHF as contributing factors to the care gaps include shortage of healthcare professionals with adequate training in $\mathrm{AF}$ management including ECG interpretation and initiation of anticoagulation therapy, difficulty accessing health care particularly by those who live in rural areas, reluctance of physicians and patients to initiate oral anticoagulation therapy, and non-adherence to treatment due to lack of access to anticoagulants or lack of awareness of the importance of medication adherence (19).

In addition, although warfarin is relatively cheaper compared to non-vitamin $\mathrm{K}$ antagonist oral anticoagulants (NOACs), most resource limited settings don't have mechanisms for safe INR monitoring (6). In GARFIELDAF, $36.9 \%$ of patients with $\mathrm{CHA}_{2} \mathrm{DS}_{2-}$ VASC score $\geq 2$ did not receive anticoagulation (20). A prospective study of 172 patients in Cameroon also showed low rates of anticoagulation at $34.5 \%$ (21). Studies from Latin America and other sub-Saharan countries have consistently shown highly variable but overall low rates of anticoagulation for AF (7-10). The rates of anticoagulation in patients with $\mathrm{CHA}_{2} \mathrm{DS}_{2}$ VASC $\geq 2$ from the RE-LY study ranged from $65.7 \%$ in North America to only $11.2 \%$ in China and the mean time in therapeutic range was $40 \%$ or less in India, China, Southeast Asia, and Africa (12). Ongoing studies such as the investigation of rheumatic $\mathrm{AF}$ treatment using vitamin $\mathrm{K}$ antagonists, rivaroxaban or aspirin studies (INVICTUS), which compares rivaroxaban to warfarin in patients with rheumatic heart disease related $\mathrm{AF}$, will help generate evidence regarding the role of NOACs in rheumatic valvular $\mathrm{AF}$ (22).

There is also very limited access to invasive management of AF including catheter ablation in LMICs due to the high cost of the procedures and inadequate number of trained experts. A study from 31 countries in Africa on rates of interventional electrophysiological procedures shows only 8 countries provided consistent catheter ablation therapies mainly under the supervision of visiting specialists (23). The gross underutilization of interventional electrophysiological services in Africa was attributed to high procedural costs, lack of facilities and trained physicians, and shortage of specialty training programs (23).

\section{Future directions}

The key to improving AF care in LMIC requires the establishment of strong CVD health system to ensure sustainability. Structural interventions including training health care workers adequately, strengthening the diagnostic and treatment capabilities of healthcare facilities and emergency response system is important. The evidence-base for the applicability of current international AF management guidelines to LMICs, identifying costeffective interventions and how to successfully implement them in resource limited settings needs to be well studied and understood. Potential solutions recommended by the WHF's AF roadmap as part of the World Health Organization's (WHO) Global Action Plan Targets to reduce premature deaths from non-communicable diseases by $25 \%$ by 2025 include training low level providers to screen for AF, using telemedicine technologies for diagnosis and management, developing country-specific health care worker and patient level training and education, and developing simplified and locally applicable AF guidelines (19). To improve anticoagulation rates, the WHF recommends training non-cardiologist and non-physician healthcare providers to manage anticoagulation therapy, researching the feasibility of self-monitoring programs for patients on anticoagulation, and providing universal coverage for essential medicines including anticoagulation for $\mathrm{AF}(19)$.

\section{Bradyarrhythmias}

\section{Epidemiology}

The epidemiology of SND and atrioventricular (AV) block has not been well studied in LMICs. In the last world survey on cardiac pacing and implantable cardioverterdefibrillator (ICD) implantation in 2011, more than 100 LMICs did not report any data and only 2 African countries (South Africa and Sudan) participated (24). Limited data from North African countries are included in the European Heart Rhythm Association (EHRA) White Book which summarises cardiac implantable electronic devices (CIED) statistics from most European Society of Cardiology (ESC) countries (25). In 2017, the Pan African Society of 


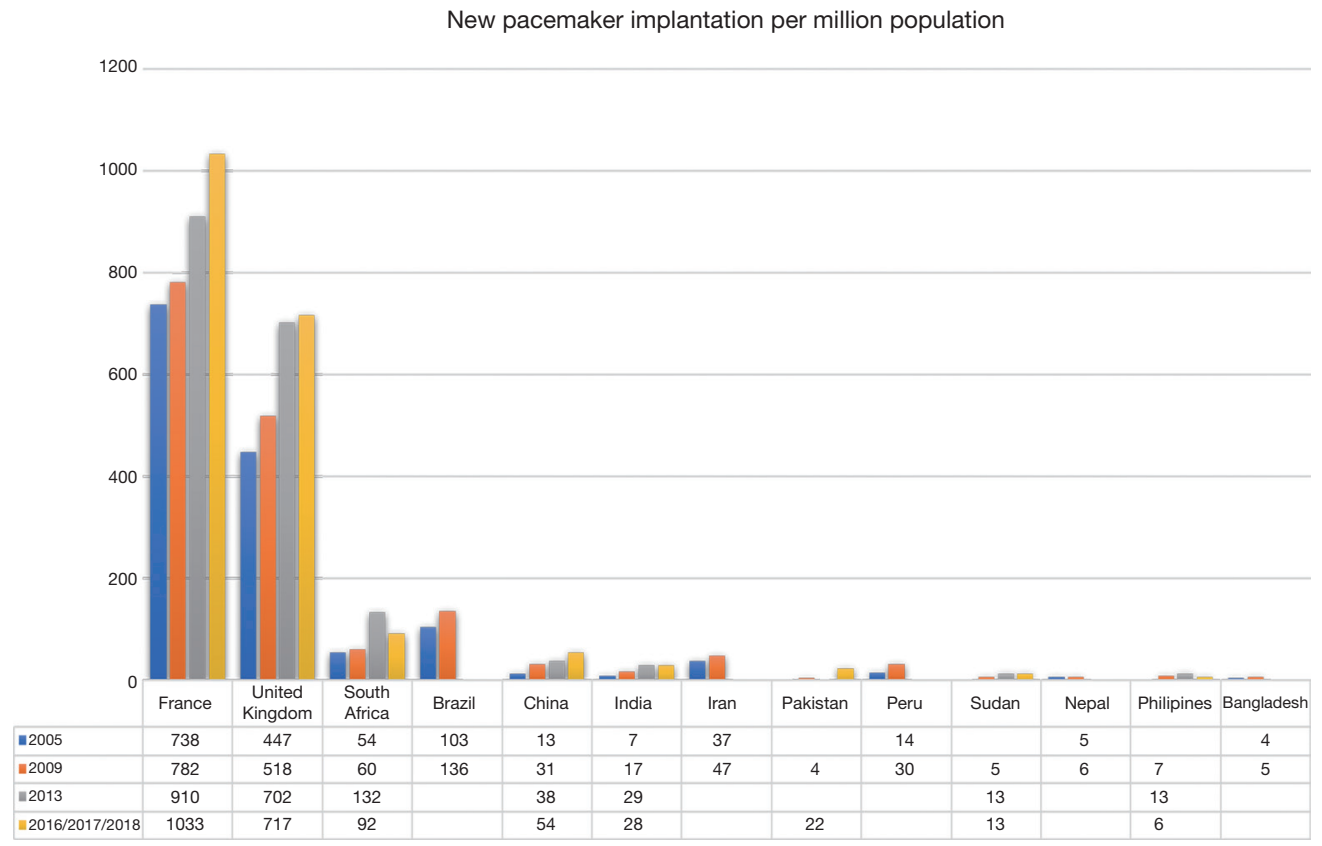

Figure 1 New pacemaker implants per million population in 2005, 2009, 2013, 2017 and 2018 according to the 11th world survey of cardiac pacing and Implantable Cardiaveter Defibrilators, The Cardiac Arrhythmia and Pacing Task Forces of the Pan-African Society of Cardiology (PASCAR) report in 2018, European Heart Rhythm Association (EHRA) report in 2015 and the Asia Pacific Heart Rhythm Society (APHRS) white book of 2018 (23,25-29).

Cardiology (PASCAR) published a report of 2011-2016 statistics on the use of CIED and electrophysiological procedures in 31 African countries (23). This report found that more than $20 \%$ of SSA countries did not have a permanent pacemaker implantation facility, needed humanitarian foreign missions to treat AV block or needed to send patients to other countries for permanent pacemakers. Marked variation in cost (up to 1,000 -fold) was observed across countries with the mean cost exceeding the average monthly earning in more developed SSA countries, making this financial aspect the main barrier for treating such patients. A second report has recently been published updating CIED and electrophysiological procedures up to 2018 (26). The operator density (a median of 0.158 operators per million population per country), rate of pacing centers which was $<1$ per million population and the implantation rate ( 2.78 per million inhabitants) did not significantly improve over the 2-year follow-up period, maintaining a $>200$-fold gap with the average implantation rates of a western European country.

In the 11 th world survey of cardiac pacing, Asian countries (Bangladesh, Indonesia, Pakistan, the Philippines, Vietnam, Nepal to name but a few) claimed less than 10 new implants per million population whereas Germany, France, and United States had more than 750 per million population (Figure 1) (25). In real world data from India and Bangladesh in 2017, it was reported that only 1 in 6 patients with SND received pacemaker indicated therapy (30). This finding is a common scenario in the majority of LMICs (24). In the 2018 PASCAR survey, Tunisia (164.3 implants per million), Mauritius (150.2 implants per million), and South Africa (92.1 implants per million) had the highest implantation rates (26).

\section{Rationale for national registries}

Although the importance of national device registries has been recognised and accepted, there is lack of consensus on how to establish national device registries. Registries provide a 'real-world' picture for patients, physicians, manufacturers, funders, decision-makers and other stakeholders. In this context, medical device registries are important for regulatory decisions, establishing safety and approval of the medical device. For funders, medical device registries provide evidence of benefit of the medical device and drive the decision whether the product should be 
reimbursed or not. For hospitals, data from medical device registries are important for sound procurement decisions, and last - and of paramount importance-medical device registries help patients and their physicians make joint decisions on which product is the most appropriate (24). In LMICs, device registries are non-existent apart from 1 single center registry in Nigeria (24). The rest of African countries did not have any national or single center registries up to 2018 (26). Therefore, statistics on the use of pacemakers in LMICs are mainly based on self-reporting which is inaccurate.

\section{Reconditioned devices}

Reutilization of pacemakers has been performed for more than 3 decades with the support of 1985 North American Society of Pacing and Electrophysiology Policy Conference and the 2002 American College of Cardiology/American Heart Association/ North American Society of Pacing and Electrophysiology Guidelines Update for implantation of cardiac pacemakers $(31,32)$. Although numerous studies have shown that pacemaker reuse is safe and effective (33-35), the rate of reused pacemaker implantations has not increased as expected (23). In Africa, approximately $20 \%$ of countries implant reused pacemakers; however, details on how many reused pacemakers are implanted in these countries are unknown. In the 2018 PASCAR survey, only Sierra Leone reported a 1:1 ratio of reused versus new devices (23). This relatively high rate of pacemaker reuse is explained by the fact that this country is actively involved in a study on reuse device safety and efficacy conducted by the US organization My Heart Your Heart.

\section{Challenges to pacing in LMICs}

The aging population is growing in the majority of LMICs and the indications for pacemaker implantation is expected to increase. However, this expected increase in pacemaker implantations is not matched by the current very low rate of pacemaker implantations in almost all SSA countries and many other LMICS such as Bangladesh, Indonesia, Pakistan, the Philippines, Vietnam, Nepal which had $<10$ implants per million population in the last decade. Several barriers of the access to device implantations have been identified (25).

Visiting philanthropic missions have been successful to assist LMICs without pacing facilities. Project Pacer International has worked in Bolivia for more than 3 decades and has expanded its services to India and other countries in South America and Africa (36). The French organization "Cercle de Rythmologie Africain" have performed many pacemaker implantations in French-speaking countries (36). Pace for Life (UK) and My Heart Your Heart (Michigan, USA) are 2 NGOs working closely with PASCAR in Africa. They have proposed more sustainable support in training local physicians in Sierra Leone, Sudan, Ethiopia, Zambia, and Kenya (33). Training locally based physicians in the techniques of cardiac pacing and electrophysiology remains the most sustainable and cost-effective way to provide sustainable arrhythmia care in LMICs. Many doctors from LMICs are being trained in countries like India, China, Tunisia, Morocco, Algeria, Sudan, and South Africa. Having local physicians trained in pacemaker follow-up has advantages over philanthropic missions in that pacemaker follow-up problems (like sepsis, lead repositioning, suitable pacemaker programming) can be managed effectively and does not rely on external help.

In Africa, the cost of single-chamber pacemaker procedure ranges from $\$ 690$ to $\$ 3,500$, which is more than the average monthly earnings in more developed African countries. Thus, in the context of out-of-pocket healthcare system, cost of implantations is regarded as the main barrier to expand this activity (23). With an estimated need of about 1 million pacemakers per year, the gap between supply and demand remains wide (37).

\section{Supraventricular tachyarrhythmias}

\section{Epidemiology}

Supraventricular tachycardias (SVT) are tachycardias (atrial or ventricular rate greater than 100 beats per minute) that need the atrial tissue or the $\mathrm{AV}$ nodal tissue for initiation or maintenance $(38,39)$. Data from HICs have reported the prevalence of paroxysmal SVT in the general population to be 2.25 per 1,000 persons, and the incidence is approximately 35 per 100,000 person-years (40). There are limited epidemiological studies of SVTs from LMICs.

\section{Challenges to management of SVT in LMICs}

Pharmacological treatment of recurrent SVT has been found to be more costly than curative catheter ablation (41). However, because of lack of expertise for diagnostic electrophysiological studies and catheter ablation and the high initial cost of the ablation particularly when $3 \mathrm{D}$ 
mapping systems are used, medical therapy is often firstline and the mainstay strategy for the management of SVT in LMICs (36). Catheter ablation is feasible for most SVTs with high curative rates and low SVT recurrence (42). Thus, in high-income countries catheter ablation remains the treatment of choice for recurrent SVT (39).

The perceived excessive cost of catheter ablation may be one of the reasons for the delay in the emergence of this technique in most LMICs. SVTs result in a substantial number of emergency room visits, most which end up in hospital admissions. Further, catheter ablation is curative for most SVTs. Some ablation catheters can be reused after appropriate resterilization up to five times without losing mechanical and electrical integrity and no risk of microbial contamination, thus curbing the cost $(43,44)$. In a cost effectiveness analysis performed in Guatemalan adults' patients with SVT, Rodriguez et al. found that the cost of catheter ablation was $\$ 5,411$. Catheter ablation lead to 1.46 quality adjusted life years (QALYs) gain and was $\$ 7,993$ cheaper than medical therapy, which represented a saving of $\$ 5,480$ per QALY gained (41).

A contemporary PASCAR report confirmed that only 5 of 23 countries surveyed (South Africa, Algeria, Kenya, Tunisia and Senegal) were able to offer atrial flutter ablations, accessory pathway ablations and ablation for AV nodal reentrant tachycardia (26). Only 4 countries (South Africa, Algeria, Egypt and Tunisia) were able to offer complex ablation using a $3 \mathrm{D}$ mapping system. Access to catheter ablation is variable in LMICs. In 2006, catheter ablations were performed in up to 20,000 cases with a more than $98 \%$ success for rate for atrioventricular nodal reentry tachycardia (AVNRT) and atrioventricular reentry tachycardia (AVRT) in China (45). The number of centers with access to 3D mapping in China is increasing (45). Similarly, the number of centers with cardiac electrophysiology services in India has been increasing, from 4 centers in 1995 to approximately 400 in 2006, albeit, mostly in the private sector (46). Consequently, the number of catheter ablations procedures performed in India has also increased (46). Similarly, the number of curative catheter ablations procedures performed in Nepal has steadily increased over the years despite challenges (47). The Latin American Society of Electrophysiology and Cardiac Stimulation (SOLAECE) registry was a retrospective registry that assessed the use of catheter ablation in Latin America over a 12-month period (48). The SOLAECE registry received data from 120 centers in 13 countries which represent $90 \%$ of the Latin American population (48). Around 79\% of the procedures reported by the SOLAECE registry were performed in two countries (Brazil and Argentina) and mostly in the private sector. A dedicated cardiac electrophysiology laboratory was only present in $60 \%$ of the SOLAECE centers and 3D mapping available in $49 \%$ (48). Eighty-five percent of the SOLAECE registry ablation were for SVTs and the success rate was high (92\%) with a low complication rate (4\%) (48).

Electrophysiological studies and ablations during medical missions are still occurring but do not provide a sustainable long-term solution. Sok-Sithikun and colleagues performed 31 radiofrequency ablations of different types of SVTs during medical missions to Morocco with a $93.5 \%$ success rate. Although the procedures were performed by experienced cardiac electrophysiologists, they were performed in orthopedics or gynecology theater, without good quality fluoroscopy and contemporary electroanatomic mapping or ablation catheters (49).

\section{Sudden cardiac death}

\section{Epidemiology}

Most epidemiological studies of sudden cardiac death (SCD) are from HICs. In the United States, SCD claims up to 300,000 lives annually (50). The reported incidence of SCD in Europe and North America ranges between 50 and 100 per 100,000 in the general population (51). Ventricular tachyarrhythmias [ventricular tachycardia (VT) and ventricular fibrillation (VF)] are the most common mechanisms of SCD (52,53). In 157 patients who suffered SCD while wearing a Holter monitor, $84 \%$ had a ventricular tachyarrhythmia (VT/VF) and $16 \%$ had a bradyarrhythmia as the cause of SCD (52).

Epidemiological studies of SCD from Africa are mostly lacking. There are limited prospective data on the incidence and prevalence of SCD from Africa; in this regard, most of the studies in LMIC are necropsy studies or hospital based studies (54-57). Emergency medical services are limited on most LMICs and therefore data on the true incidence of SCD remains difficult to determine. The Douala SCD study prospectively assessed the incidence of the SCD in Douala, Cameroon: the authors reported a crude incidence rate of SCD of 31.3 per 100,000 person-years (58). In the Douala SCD study the risk of SCD increased with increasing age except for the 30- to 39-year-old age group, the distribution linearly and steadily increased with age and peaked in those older than 69 years (58). A study from South Africa investigated out of 
hospital SCD based only on data from first responders and reported an incidence of SCD of 6.4 per 100,000 personyears which is likely an underestimate (59). A prospective study to determine the incidence of SCD in China was performed in 4 regions with disparate socioeconomic status: a total of 678,718 subjects were monitored for 12 months, 284 SCDs occurred (60). The overall incidence of SCD was 41.8 per 100,000 (60). Regional variations in the incidence of SCD were detected, with higher rates in rural communities with low socioeconomic status (60). Using a mailed questionnaire and direct retrospective interview of relatives or witness, Tungsanga and Sriboolue reported an incidence of SCD in adults aged 20-49 years old of 38 per 100,000 in North East Thailand (61). Data from India is scanty and one study reported that SCD accounts for $10 \%$ of all total deaths (62).

The main cause of SCD in HICs is atherosclerotic coronary artery disease and accounts for up to $60 \%$ of SCD (63). The reported causes of SCD in LIMCs are heterogenous. In a necropsy study by Schneider and Bezabih in Addis Ababa, Ethiopia, coronary artery disease was found to be the leading cause of SCD (54). Additionally, in an autopsy study from South India, Srivatsa and colleagues reported that myocardial infarction was responsible for $87 \%$ of SCD (64). However, in an autopsy study from Nigeria by Rotimi et al., coronary artery disease accounted for only $4 \%$ of SCD and hypertensive heart disease was the leading cause of SCD (55). Reports from HICs indicate that congenital anomalies of coronary arteries, myocarditis and hypertrophic cardiomyopathy account for large proportion of SCD individuals younger than 35 years with structurally abnormal hearts $(65,66)$. Similarly, in an autopsy study of individual who suffered SCD during a sports activity, Allouche et al. from Tunisia found that among those younger than 35 years of age hypertrophic cardiomyopathy accounted for $41 \%$ of SCD and arrhythmogenic right ventricular cardiomyopathy/ dysplasia accounted for 13.6\% (67). The PASCAR Sudden Cardiac Death Survey is a prospective registry that will evaluate the incidence, prevalence, cause and outcomes of SCD in numerous African countries $(68,69)$. Similar registries are needed in other LMICs.

\section{Challenges to management of SCD in LMICs}

In both primary prevention and secondary prevention trials, ICDs show important mortality benefits (70-74). Despite recommendations by cardiac societies, considerable challenges face LIMCs regarding access to and implantation of ICDs including the high cost of the devices, lack of facilities and expertise to implant them. For example, $18 \%$ of the SSA countries do not have a registered cardiologist, 11 of 33 (33\%) PASCAR countries do not perform pacemaker implantations and ICDs (75). The Improve SCA study which mainly enrolled patients from Asia, Latin America, Eastern Europe, the Middle East and Africa showed that only $40 \%$ of clinically indicated patients undergo ICD implantation (76). In addition, $46 \%$ and $10 \%$ of patients with a primary prevention and secondary prevention indications for an ICD respectively, refuse ICD implantation (76). The highest refusal rates for primary prevention and secondary prevention are in India (77\%) and Singapore (26\%) respectively (76). The high monetary cost of ICDs and the patient's perceived lack of benefit from the ICD were the main reasons for refusal of ICD implantation (76). An ICD generator price is estimated to between $\$ 20,000$ and $\$ 40,000$ and ICD leads cost more than $\$ 10,000$ (33). Further, in most African countries and other LMICs health care costs (up to 56\%) are paid out of pocket by household incomes (77). The lack of efficacy of antiarrhythmic drugs in the prevention and treatment of sudden cardiac death is particularly relevant in LMICs where drug therapy may be incorrectly seen as an alternative over proven device therapy (74). Cardiac sympathetic denervation is an effective treatment in patients with refractory ventricular arrhythmias with patients with channelopathies and cardiomyopathies. This treatment option is currently being tested to prevent ventricular arrhythmias in a randomized trial in South Africa (78).

Regional and global efforts to improve the management of arrhythmias are ongoing. For example, the PASCAR pacing fellowship is training physicians and technologist to implant and follow-up pacemakers in underserved SSA countries (33); resterilisation of used pacemakers and ICDs has been shown to be safe and feasible, thus curbing the costs associated with new devices (35); finally, out pocket payments for health care are on a modest decline, perhaps in the future this form of therapy will be accessible with limited out of pocket payment (77).

\section{Conclusions}

The burden of cardiac arrhythmias is expected to increase in LMICs. There is a paucity of data on the epidemiology of cardiac arrhythmias particular in SSA. Guidelinedirected management of cardiac arrhythmias in LMICs is 
challenging because of the paucity of skilled personnel, lack of basic infrastructure, and the lack of device and ablative therapy for the treatment of cardiac arrhythmias.

\section{Acknowledgments}

Funding: None.

\section{Footnote}

Provenance and Peer Review: This article was commissioned by the Guest Editor (Ntobeko A. B. Ntusi) for the series "Cardiovascular Diseases in Low- and Middle-Income Countries" published in Cardiovascular Diagnosis and Therapy. The article was sent for external peer review organized by the Guest Editor and the editorial office.

Conflicts of Interest: All authors have completed the ICMJE uniform disclosure form (available at http://dx.doi. org/10.21037/cdt.2019.09.21). The series "Cardiovascular Diseases in Low-and Middle-Income Countries" was commissioned by the editorial office without any funding or sponsorship. The authors have no conflicts of interest to declare.

Ethical Statement: The authors are accountable for all aspects of the work in ensuring that questions related to the accuracy or integrity of any part of the work are appropriately investigated and resolved.

Open Access Statement: This is an Open Access article distributed in accordance with the Creative Commons Attribution-NonCommercial-NoDerivs 4.0 International License (CC BY-NC-ND 4.0), which permits the noncommercial replication and distribution of the article with the strict proviso that no changes or edits are made and the original work is properly cited (including links to both the formal publication through the relevant DOI and the license). See: https://creativecommons.org/licenses/by-nc-nd/4.0/.

\section{References}

1. Gersh BJ, Sliwa K, Mayosi BM, et al. The epidemic of cardiovascular disease in the developing world: global implications. Eur Heart J 2010;31:642-8.

2. Organisation WH. Cardiovascular diseases (CVDs) Key Facts. Available online: https://www.who.int/en/newsroom/fact-sheets/detail/cardiovascular-diseases-(cvds).
Accessed 16 June 2019.

3. Chugh SS, Roth GA, Gillum RF, et al. Global Burden of Atrial Fibrillation in Developed and Developing Nations. Global Heart 2014;9:113-9.

4. Patel NJ, Atti V, Mitrani RD, et al. Global rising trends of atrial fibrillation: a major public health concern. Heart 2018;104:1989.

5. (CDC) CfDCaP. Atrial Fibrillation Fact Sheet. 2017. Available online: https://www.cdc.gov/dhdsp/data statistics/fact_sheets/fs_atrial_fibrillation.htm. Accessed July 212019.

6. Rahman F, Kwan GF, Benjamin EJ. Global epidemiology of atrial fibrillation. Nat Rev Cardiol 2014;11:639-54.

7. Bai Y, Wang YL, Shantsila A, et al. The Global Burden of Atrial Fibrillation and Stroke: A Systematic Review of the Clinical Epidemiology of Atrial Fibrillation in Asia. Chest 2017;152:810-20.

8. Marcolino MS, Palhares DM, Benjamin EJ, et al. Atrial fibrillation: prevalence in a large database of primary care patients in Brazil. Europace 2015;17:1787-90.

9. Cubillos L, Haddad A, Kuznik A, et al. Burden of disease from atrial fibrillation in adults from seven countries in Latin America. Int J Gen Med 2014;7:441-8.

10. Stambler BS, Ngunga LM. Atrial fibrillation in SubSaharan Africa: epidemiology, unmet needs, and treatment options. Int J Gen Med 2015;8:231-42.

11. Morillo CA, Banerjee A, Perel P, et al. Atrial fibrillation: the current epidemic. J Geriatr Cardiol 2017;14:195-203.

12. Oldgren J, Healey JS, Ezekowitz M, et al. Variations in cause and management of atrial fibrillation in a prospective registry of 15,400 emergency department patients in 46 countries: the RE-LY Atrial Fibrillation Registry. Circulation 2014;129:1568-76.

13. Healey JS, Oldgren J, Ezekowitz M, et al. Occurrence of death and stroke in patients in 47 countries 1 year after presenting with atrial fibrillation: a cohort study. Lancet 2016;388:1161-9.

14. Bassand JP, Accetta G, Camm AJ, et al. Two-year outcomes of patients with newly diagnosed atrial fibrillation: results from GARFIELD-AF. Eur Heart J 2016;37:2882-9.

15. January CT, Wann LS, Alpert JS, et al. 2014 AHA/ACC/ HRS guideline for the management of patients with atrial fibrillation: executive summary: a report of the American College of Cardiology/American Heart Association Task Force on practice guidelines and the Heart Rhythm Society. Circulation 2014;130:2071-104.

16. January CT, Wann LS, Calkins H, et al. 2019 AHA/ ACC/HRS Focused Update of the 2014 AHA/ACC/ 
HRS Guideline for the Management of Patients With Atrial Fibrillation: A Report of the American College of Cardiology/American Heart Association Task Force on Clinical Practice Guidelines and the Heart Rhythm Society. J Am Coll Cardiol 2019;74:104-32.

17. Kirchhof P, Benussi S, Kotecha D, et al. 2016 ESC Guidelines for the management of atrial fibrillation developed in collaboration with EACTS. Eur Heart J 2016;37:2893-962.

18. Granger CB, Alexander JH, McMurray JJ, et al. Apixaban versus Warfarin in Patients with Atrial Fibrillation. N Engl J Med 2011;365:981-92.

19. Murphy A, Banerjee A, Breithardt G, et al. The World Heart Federation Roadmap for Nonvalvular Atrial Fibrillation. Glob Heart 2017;12:273-84.

20. Kakkar AK, Mueller I, Bassand JP, et al. Risk Profiles and Antithrombotic Treatment of Patients Newly Diagnosed with Atrial Fibrillation at Risk of Stroke: Perspectives from the International, Observational, Prospective GARFIELD Registry. PLoS One 2013;8:e63479.

21. Ntep-Gweth M, Zimmermann M, Meiltz A, et al. Atrial fibrillation in Africa: clinical characteristics, prognosis, and adherence to guidelines in Cameroon. Europace 2010;12:482-7.

22. INVestIgation of rheumatiC AF Treatment Using Vitamin K Antagonists, Rivaroxaban or Aspirin Studies, Superiority (INVICTUS-ASA. Available online: https://clinicaltrials. gov/ct2/show/NCT02832531\#contacts

23. Bonny A, Ngantcha M, Jeilan M, et al. Statistics on the use of cardiac electronic devices and interventional electrophysiological procedures in Africa from 2011 to 2016: report of the Pan African Society of Cardiology (PASCAR) Cardiac Arrhythmias and Pacing Task Forces. Europace 2017;20:1513-26.

24. Zhang S, Gaiser S, Kolominsky-Rabas PL. Cardiac implant registries 2006-2016: a systematic review and summary of global experiences. BMJ Open 2018;8:e019039.

25. Mond HG, Proclemer A. The 11th World Survey of Cardiac Pacing and Implantable CardioverterDefibrillators: Calendar Year 2009-A World Society of Arrhythmia's Project. Pacing Clin Electrophysiol 2011;34:1013-27.

26. Presented at the Late Breaking Sessions, European Society of Cardiology/World Heart Federation, Paris, France, 3 September 2019.

27. Raatikainen MJ, Arnar DO, Zeppenfeld K, et al. Statistics on the use of cardiac electronic devices and electrophysiological procedures in the European Society of Cardiology countries: 2014 report from the European Heart Rhythm Association. Europace 2015;17 Suppl $1: \mathrm{i} 1-75$.

28. Societe APHR. Asia Pacific Heart Rhythm Societe White Book. 2018. Accessed 18 Sept 2019.

29. Raatikainen MJ, Arnar DO, Merkely B, et al. A Decade of Information on the Use of Cardiac Implantable Electronic Devices and Interventional Electrophysiological Procedures in the European Society of Cardiology Countries: 2017 Report from the European Heart Rhythm Association. Europace 2017;19:1i1-90.

30. Narasimhan C, Sanyal J, Sethi R, et al. Underutilization of pacemaker therapy for sinus node dysfunction - Real world data from South Asia. Indian Heart J 2017;69:607-12.

31. Boal BH, Escher DJ, Furman S, et al. Report of the policy conference on pacemaker reuse sponsored by the North American Society of Pacing and electrophysiology. J Am Coll Cardiol 1985;5:808-10.

32. Gregoratos G, Abrams J, Epstein AE, et al. ACC/AHA/ NASPE 2002 Guideline Update for Implantation of Cardiac Pacemakers and Antiarrhythmia Devices. J Am Coll Cardiol 2002;40:1703-19.

33. Sani MU, Mayosi BM. The Pacemaker and ICD Reuse Programme of the Pan-African Society of Cardiology. Heart 2017;103:1844.

34. Nava S, Morales JL, Marquez MF, et al. Reuse of pacemakers: comparison of short and long-term performance. Circulation 2013;127:1177-83.

35. Jama ZV, Chin A, Badri M, et al. Performance of re-used pacemakers and implantable cardioverter defibrillators compared with new devices at Groote Schuur Hospital in Cape Town, South Africa. Cardiovasc J Afr 2015;26:181-7.

36. Bestawros M. Electrophysiology in the Developing World: Challenges and Opportunities. Cardiol Clin 2017;35:49-58.

37. Mond HG, Mick W, Maniscalco BS. Heartbeat International: Making "poor" hearts beat better. Heart Rhythm 2009;6:1538-40.

38. Delacrétaz E. Supraventricular Tachycardia. N Engl J Med 2006;354:1039-51.

39. Page RL, Joglar JA, Caldwell MA, et al. 2015 ACC/ AHA/HRS Guideline for the Management of Adult Patients With Supraventricular Tachycardia. Circulation 2016;133:e506-74.

40. Orejarena LA, Vidaillet H, DeStefano F, et al. Paroxysmal Supraventricular Tachycardia in the General Population. J 
Am Coll Cardiol 1998;31:150-7.

41. Rodriguez BC, Leal S, Calvimontes G, et al. CostEffectiveness of Radiofrequency Ablation for Supraventricular Tachycardia in Guatemala: Patient Outcomes and Economic Analysis from a Low- to MiddleIncome Country. Value Health Reg Issues 2015;8:92-8.

42. Jackman WM, Beckman KJ, McClelland JH, et al. Treatment of Supraventricular Tachycardia Due to Atrioventricular Nodal Reentry by Radiofrequency Catheter Ablation of Slow-Pathway Conduction. N Engl J Med 1992;327:313-8.

43. Avitall B, Khan M, Krum D, et al. Repeated use of ablation catheters: A prospective study. J Am Coll Cardiol 1993;22:1367-72.

44. Bathina MN, Mickelsen S, Brooks C, et al. Safety and efficacy of hydrogen peroxide plasma sterilization for repeated use of electrophysiology catheters. J Am Coll Cardiol 1998;32:1384-8.

45. Chen YH, Chen H, Wu Y, et al. Cardiac electrophysiology in China. Heart Rhythm 2007;4:862.

46. Francis J, Lokhandwala Y. Electrophysiology in India. Heart Rhythm 2007;4:687.

47. Rajbhandari S, Bahadur M, Naik A, et al. Electrophysiology around the world: Nepal. Heart Rhythm 2007;4:1367.

48. Keegan R, Aguinaga L, Fenelon G, et al. The first Latin American Catheter Ablation Registry. Europace 2015;17:794-800.

49. Bun SS, Latcu DG, Errahmouni A, et al. Pacemakers implantation and radiofrequency catheter ablation procedures during medical missions in Morocco: an 8-year experience. Europace 2016;18:1038-42.

50. John RM, Tedrow UB, Koplan BA, et al. Ventricular arrhythmias and sudden cardiac death. Lancet 2012;380:1520-9.

51. Fishman GI, Chugh SS, DiMarco JP, et al. Sudden cardiac death prediction and prevention: report from a National Heart, Lung, and Blood Institute and Heart Rhythm Society Workshop. Circulation 2010;122:2335-48.

52. Bayés de Luna A, Coumel P, Leclercq JF. Ambulatory sudden cardiac death: Mechanisms of production of fatal arrhythmia on the basis of data from 157 cases. Am Heart J 1989;117:151-9.

53. Watanabe E, Tanabe T, Osaka M, et al. Sudden cardiac arrest recorded during Holter monitoring: Prevalence, antecedent electrical events, and outcomes. Heart Rhythm 2014;11:1418-25.

54. Schneider J, Bezabih K. Causes of sudden death in Addis
Ababa, Ethiopia. Ethiop Med J 2001;39:323-40.

55. Rotimi O, Ajayi AA, Odesanmi WO. Sudden unexpected death from cardiac causes in Nigerians: A review of 50 autopsied cases. Int J Cardiol 1998;63:111-5.

56. Talle MA, Bonny A, Bakki B, et al. Sudden Cardiac Death: Clinical Perspectives from the University of Maiduguri Teaching Hospital, Nigeria. World J Cardiovasc Dis 2015;5:95-106.

57. Akinwusi PO, Komolafe AO, Olayemi OO, et al. Pattern of sudden death at Ladoke Akintola University of Technology Teaching Hospital, Osogbo, South West Nigeria. Vasc Health Risk Manag 2013;9:333-9.

58. Bonny A, Tibazarwa K, Mbouh S, et al. Epidemiology of sudden cardiac death in Cameroon: the first populationbased cohort survey in sub-Saharan Africa. Int J Epidemiol 2017;46:1230-8.

59. Stein C. Out-of-hospital cardiac arrest cases in Johannesburg, South Africa: a first glimpse of short-term outcomes from a paramedic clinical learning database. Emerg Med J 2009;26:670-4.

60. Hua W, Zhang LF, Wu YF, et al. Incidence of Sudden Cardiac Death in China: Analysis of 4 Regional Populations. J Am Coll Cardiol 2009;54:1110-8.

61. Tungsanga K, Sriboonlue P. Sudden unexplained death syndrome in north-east Thailand. Int J Epidemiol 1993;22:81-7.

62. Rao BH, Sastry BK, Chugh SS, et al. Contribution of sudden cardiac death to total mortality in India - a population based study. Int J Cardiol 2012;154:163-7.

63. Zheng ZJ, Croft JB, Giles WH, et al. Sudden Cardiac Death in the United States, 1989 to 1998. Circulation 2001;104:2158-63.

64. Srivatsa UN, Swaminathan K, Sithy Athiya Munavarah $\mathrm{K}$, et al. Sudden cardiac death in South India: Incidence, risk factors and pathology. Indian Pacing Electrophysiol J 2016;16:121-5.

65. Drory Y, Turetz Y, Hiss Y, et al. Sudden unexpected death in persons < 40 years of age. Am J Cardiol 1991;68:1388-92.

66. Eckart RE, Scoville SL, Campbell CL, et al. Sudden Death in Young Adults: A 25-Year Review of Autopsies in Military Recruits. Ann Intern Med 2004;141:829-34.

67. Allouche M, Boudriga N, Ahmed HB, et al. La mort subite au cours d'une activité sportive en Tunisie : à propos d'une série autopsique de 32 cas. Annales de Cardiologie et d'Angéiologie 2013;62:82-8.

68. Bonny A, Ngantcha M, Amougou SN, et al. Rationale and design of the Pan-African Sudden Cardiac Death survey: the Pan-African SCD study. Cardiovasc J Afr 
2014;25:176-84.

69. Chin A. Sudden cardiac death in Africa. Cardiovasc J Afr 2014;25:151-2.

70. Antiarrhythmics versus Implantable Defibrillators (AVID) Investigators. A Comparison of Antiarrhythmic-Drug Therapy with Implantable Defibrillators in Patients Resuscitated from Near-Fatal Ventricular Arrhythmias. N Engl J Med 1997;337:1576-83.

71. Connolly SJ, Gent M, Roberts RS, et al. Canadian Implantable Defibrillator Study (CIDS). Circulation 2000;101:1297-302.

72. Kuck KH, Cappato R, Siebels J, et al. Randomized Comparison of Antiarrhythmic Drug Therapy With Implantable Defibrillators in Patients Resuscitated From Cardiac Arrest. Circulation 2000;102:748-54.

73. Moss AJ, Hall WJ, Cannom DS, et al. Improved survival with an implanted defibrillator in patients with coronary disease at high risk for ventricular arrhythmia. Multicenter Automatic Defibrillator Implantation Trial Investigators. N Engl J Med 1996;335:1933-40.

Cite this article as: Mkoko P, Bahiru E, Ajijola OA, Bonny A, Chin A. Cardiac arrhythmias in low- and middle-income countries. Cardiovasc Diagn Ther 2020;10(2):350-360. doi: 10.21037/ cdt.2019.09.21
74. Bardy GH, Lee KL, Mark DB, et al. Amiodarone or an Implantable Cardioverter-Defibrillator for Congestive Heart Failure. N Engl J Med 2005;352:225-37.

75. Talle MA, Bonny A, Scholtz W, et al. Status of cardiac arrhythmia services in Africa in 2018: a PASCAR Sudden Cardiac Death Task Force report. Cardiovasc J Afr 2018;29:115-21.

76. Singh B, Zhang S, Ching CK, et al. Improving the utilization of implantable cardioverter defibrillators for sudden cardiac arrest prevention (Improve SCA) in developing countries: Clinical characteristics and reasons for implantation refusal. Pacing Clin Electrophysiol 2018;41:1619-26.

77. Xu K, Soucat A, Kutzin J, et al. Public Spending on Health: A Closer Look at Global Trends. World Health Organization 2018:58.

78. Chin A, Ntsekhe M, Viljoen C, et al. Rationale and design of a prospective study to assess the effect of left cardiac sympathetic denervation in chronic heart failure. Int J Cardiol 2017;248:227-31. 